\title{
CARDIAC COMPLICATIONS IN DUCHENNE MUSCULAR DYSTROPHY IN CHILDREN
}

\author{
Ioana Grigore', Mariana Frasin', Georgeta Diaconu' \\ ${ }^{1}$ Pediatric Neurology Clinic, "Sf. Maria”" Emergency Clinical Hospital for Children, Iasi \\ ${ }^{2}$ Functional exploration laboratory, "Sf. Maria” Emergency Clinical Hospital for \\ Children, Iasi
}

\begin{abstract}
Cardiac complications are frequently diagnosed in Duchenne muscular dystrophy (DMD), clinical manifestations generally appear after age 10. ECG recordings objectified various changes in these patients, the most common being represented by extensive $R$ waves in $V 1$, left deep $Q$ waves, conduction abnormalities and arrhythmias. Echocardiographic examination may objective the presence of subclinical cardiac dysfunction in children under 12 years old diagnosed with DMD. In patients where standard echocardiographic examination is normal, myocardial performance index is a parameter useful in early detection of asymptomatic cardiac abnormalities. Cardiovascular magnetic resonance provides information both on left ventricular systolic function and myocardial tissue changes and occurrence of fibrosis, lesions rarely objectified before the age of 10 years. All these explorations should be considered in children with DMD at the end of a complete neurological exam.
\end{abstract}

Keywords: cardiac manifestations, Duchenne muscular dystrophy, child

Progressive muscular dystrophies include a heterogeneous group of disorders characterized by primitive, progressive muscle degeneration genetically determined. Duchenne muscular dystrophy is the most common and most serious of these disorders with an incidence of 1 in 3500 newborns (Santos, 2010). Duchenne muscular dystrophy (DMD) is transmitted X-linked and is caused by the absence of dystrophin, the responsible gene that was identified in 1986 and is located on the short arm of chromosome Xp21 (Kunkel 1986, Monaco 1986).

Dystrophin normally plays an important role in stabilizing the cellular membrane both skeletal muscle and cardiac myocytes and it's absence results fragility of the sarcolemma and degeneration of muscle fibers (Menke, 1995). Pasternak (1995) noted that the decrease or absence of dystrophin causes atrophic changes at the level of muscle fiber which is dependent of the degree of deficit.

Clinically DMD is characterized by progressive decrease in muscle strength initially proximal, with worsening of motor deficit in the second and third childhood. The patients lose of ambulation by the age of 10-12 when the child requires wheelchair. Evolution of the disease is progressive, in time being affected all skeletal, respiratory and heart muscles and gastrointestinal smooth muscle.

Recent studies have reported a 90\% incidence of cardiac changes at pediatric age patients with DMD (Holmgren, 2003; Connuck, 2008). Asymptomatic cardiac involvement has been reported in $25 \%$ of children younger than 6 years and in $60 \%$ children aged 6 to 10 years (Bushby, 2003; Finsterer, 2003). McNally (2007) believes that nearly all patients with DMD who reach the third decade of life, present cardiomyopathy, but frequently diagnosis are delayed because physical inactivity of the patients masks the symptoms of heart failure. Clinical manifestations become obvious after the age of 10 years, being present in all DMD patients older than 18 years (Bushby, 2003; Finsterer, 2003). The presence at the clinical examination of some minor signs as sinus tachycardia may suggest early cardiac damage (Gulati, 2005), but usually clinical signs of cardiomyopathy appear late.

DMD associated cardiomyopathy may be considered a form of "heart dystrophy" characterized 
by impaired of architecture of myocardial cells secondary fibrosis. According to studies by nuclear magnetic resonance myocardial fibrosis is initially located at the basal-posterior and lateral wall of the left ventricle (Silva, 2007; Puchalski, 2009), the consequence being the reduction of contractile force and the disturbance of the electrical activity of myocardial cells.

\section{Electrocardiographic changes in children with Duchenne muscular dystrophy}

Electrocardiograms (ECG) have objectified in children with DMD various changes (Fig. 1, Fig. 2), the most frequently changes being represented by:

- tall R wave in V1-V2;

- deep Q wave in V5-V6;

- conduction disturbances;

- arrhythmias.

James and all (2011) considers that ECG abnormalities are common in young children diagnosed with DMD. Also they consider that heart damage is present before the onset of motor symptoms. The authors electrocardiographically analyzed 78 children under 6 years with DMD and identified ECG changes in $78 \%$ of these cases, the most common being those due to left ventricular impairment.

In a study containing a group of 131 patients with a mean age of 9 years 4 months, diagnosed with DMD, Santos and all (2010) analyzed the ECG to highlight possible changes associated with cardiac progressive muscle disease. To establish normal electrocardiographic parameters were used Garson's criteria. The authors noted that all DMD patients showed sinus rhythm at ECG recording, but at $78.6 \%$ were objectified anomalies represented by: short PR interval in $18.3 \%$ of cases, sinus tachycardia in $2.2 \%$, abnormal $\mathrm{R}$ wave in $\mathrm{V} 1$ in $29.7 \%$, abnormal Q wave in V6 in $21.3 \%$, changes in ventricular re-polarization in $54.9 \%$, right bundle branch block in 7.6\%, prolonged QT interval in $35.8 \%$, large QRS complex in $23.6 \%$, typical changes (ample R wave in V1 and deep Q wave in V6) in $6.8 \%$ of cases.

Also, Takani (2008) performed 136 ECG recordings from a group of 69 patients aged under 18 years, diagnosed with $\mathrm{DMD}$, following potential cardiac electrical changes. The author observed that $91.3 \%$ of the studied children had one or more ECG abnormalities. He also noted that $84.8 \%$ of patients younger than 10 years had ECG changes, the most common being represented by the presence of a deep Q waves, and the typical appearance described in DMD represented by ample $\mathrm{R}$ wave in V1-V5 and deep Q wave was more common in adolescents.

\section{Echocardiographic changes in children with Duchenne muscular dystrophy}

In the past at young children under the age of 12 years diagnosed with DMD is appreciated that systolic cardiac function is normal, but recent studies have echocardiographic demonstrated the presence of subclinical cardiac dysfunction in this group of patients (Mertens, 2008). Thus echocardiographic is necessary at the comprehensive evaluation of pediatric age patients with DMD. In children with

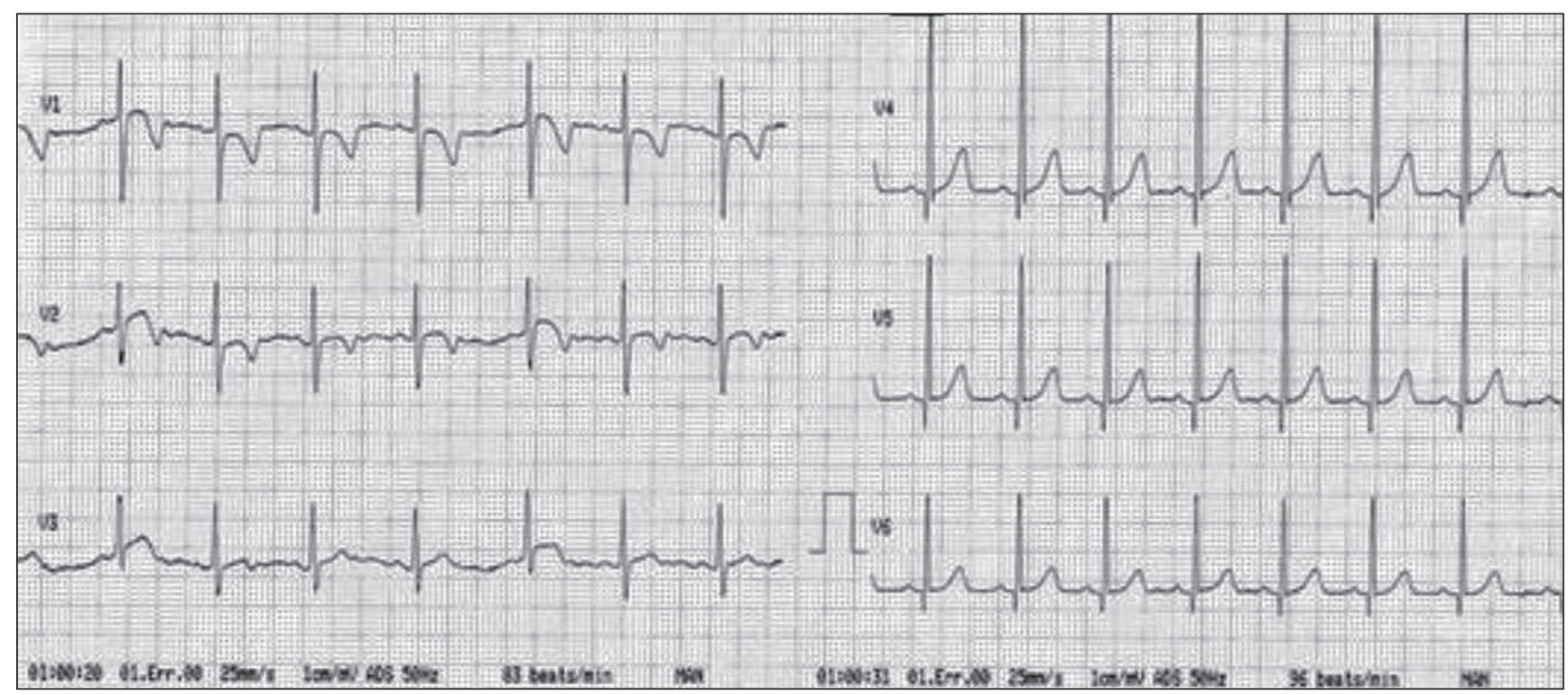

FIGURE 1. Boy of 10 years old with DMD without clinical signs of cardiac impairment. ECG: ample R wave in V1-V2 and deep Q wave in V5-V6 (Collection of Pediatric Neurology Clinic) 


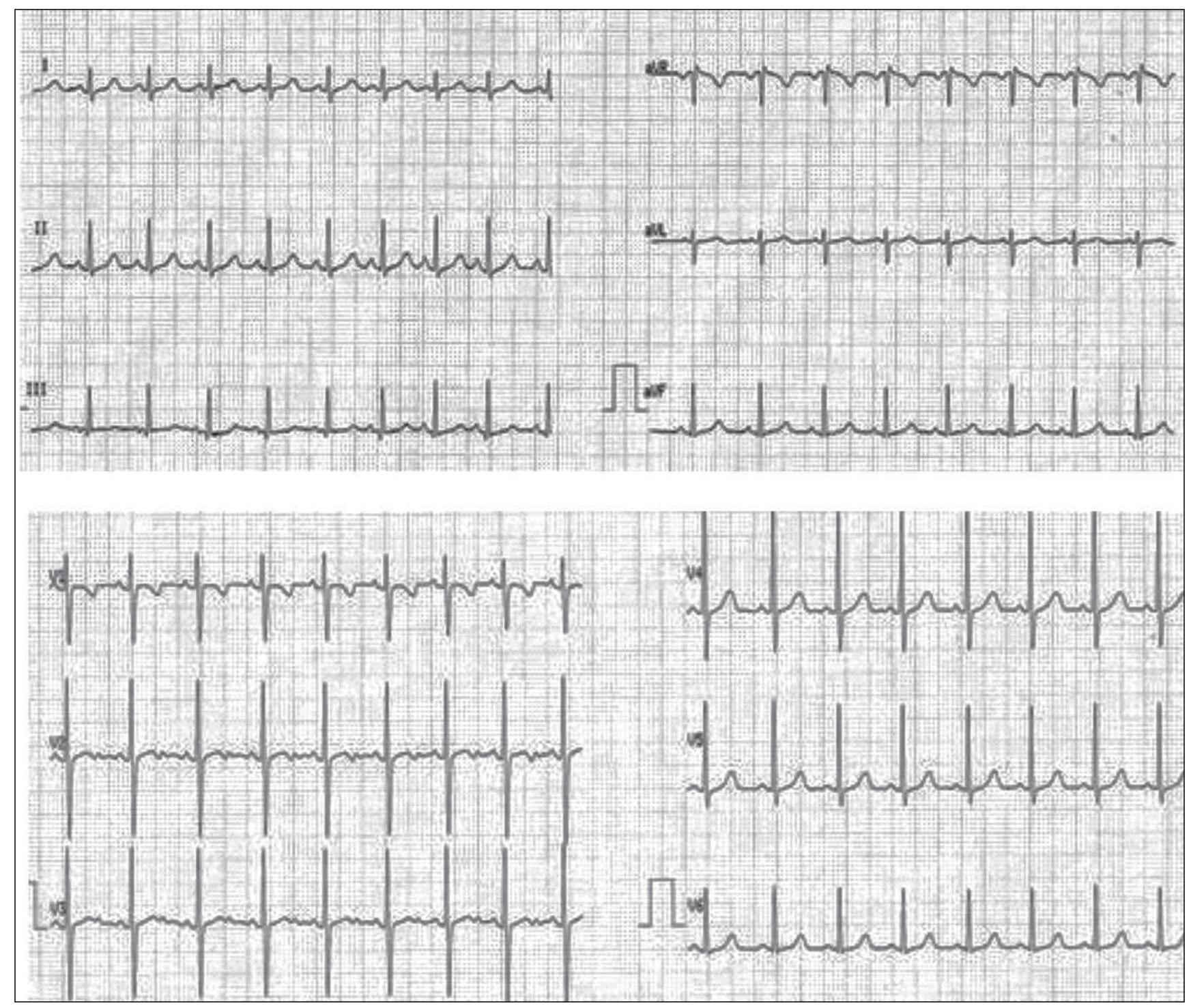

FIGURE 2. Boy of 4 years old with DMD without clinical signs of cardiac impairment. ECG: $Q T=0.35$ sec (prolonged) (Collection of Pediatric Neurology Clinic)

DMD where the echocardiographic standard examination is normal, myocardial performance index is a useful parameter in early detection of asymptomatic cardiac abnormalities (Shabanian, 2011).

Lee (2014) believes that in patients with DMD are structural and functional changes in the myocardium. The author echocardiographic explored (M-mode) 42 children with DMD and revealed atrophy in the left ventricular myocardium which was correlated with systolic function impairment and a low body mass index for age.

Gulati et al (2005) studied cardiac damage in a group of 30 children less than 6 years diagnosed with DMD with different motor impairment, 11 children having kept walking at the time of enrollment and $10 \%$ having clinical signs due to cardiac dysfunction. Approximately one third of patients were echocardiographic diagnosed with cardiomegaly, at $64.2 \%$ left ventricular ejection fraction was $<55 \%$ and at $17.8 \%$ left ventricular ejection fraction was $<50 \%$.

In another study was echocardiographic evaluated 32 children with DMD aged 3-12 years, the authors concluded that patients with DMD who had normal global systolic function can present slowdowns of diastolic myocardial contraction speed in the anterior and lateral wall of the left ventricle and in the inferolateral wall of the left ventricle (Mertens, 2008).

\section{Cardiac involvement at cardiovascular magnetic resonance in children with Duchenne muscular dystrophy}

In recent years cardiovascular magnetic resonance (CMR) is increasingly being used for diagnosis and follow of the evolution of cardiac damage in patients with DMD. Cardiovascular magnetic resonance provides informations both on left ven- 
tricular systolic function and myocardial tissue changes and occurrence of fibrosis (Florian, 2014). Cardiac lesions revealed at CMR are rarely observed before the age of 10 years (Bushby, 2010). It was also noted that the prevalence of myocardial fibrosis increases with age and can cause the decreases of the left ventricular ejection fraction (Hor, 2013). Verhaert (2011) believes that in patients with DMD the cardiomyopathy is caused by subepicardial fibrosis of the inferolateral wall, similar with the pattern observed in patients with viral myocarditis. Wansapura (2010) demonstrated at the CMR T2 signal changes, tissue edema and decreased left ventricular ejection fraction representative for micro fibrosis.

Walcher (2011) imaging cardiovascular studied a small group of 7 boys with DMD and concluded that the lesions of fibrosis are present before clinical onset of ventricular dysfunction. In turn, Bilchick (2011) assessed the prevalence and distribution of fibrotic lesions in the myocardial dysfunctional segments, on a group of 16 patients with DMD, noting that the prevalence of fibrosis at the inferior, inferolateral and anterolateral cardiac level was eight times higher than inferoseptal, anteroseptal and anterior segments.

In a study from 2013, which followed 314 patients with DMD, aged 6-28 years, Hor et al showed

\section{REFERENCES}

1. Santos M.A.B., de Assis Costa F., Travessa A.F. et al. Distrofia Muscular de Duchenne: Analisis Electrocardiografica de 131 Pacientes. Arq Bras Cardiol. 2010; 94(5):602-606.

2. Kunkel L.M. Analysis de deletions in DNA from patients with Becker and Duchenne muscular dystrophy. Nature. 1986; 322:73-7.

3. Mônaco A.P., Neve R.L., Colleti-Feener C. et al. Isolation of candidate cDNA for portions of the Duchenne muscular dystrophy gene. Nature.1986; 323:646-50.

4. Menke A., Jockusch H. Extent of shock-induced membrane leakage in human and mouse myotubes depends on dystrophim. J Cell Sci. 1995; 108:727-733.

5. Pasternak C., Wong S., Elson E.L. Mechanical function of dystrophin in muscle cells. J Cell Biol. 1995; 128:355-61.

6. Holmgren D., Wahlander H., Eriksson B.O. et al. Cardiomyopathy in children with mitochondrial disease; clinical course and cardiological findings. Eur Heart J. 2003; 24:280-8.

7. Connuck D.M., Sleeper L.A., Colan S.D. et al. Characteristics and outcomes of cardiomyopathy in children with Duchenne or Becker muscular dystrophy: a comparative study from the Pediatric Cardiomyopathy Registry. Am Heart J. 2008; 155:998-1005.

8. Bushby K. et al. Diagnosis and management of Duchenne muscular dystrophy, part 2: implementation of multidisciplinary care. Lancet Neurol. 2010; 9(2):177-89.

9. Finsterer J., Stollberger C. The heart in human dystrophinopathics. Cardiology. 2003; 99:1-19.

10. McNally E.M. New approaches in the therapy of cardiomyopathy in muscular dystrophy. Annu Rev Med. 2007; 58:75-88. lesions of cardiac fibrosis in $30 \%$ of patients to whom the left ventricular ejection fraction was $\geq$ $55 \%$ and in $84 \%$ of those in whom left ventricular ejection fraction was $<55 \%$, noting that the prevalence of cardiac damage at CMR examination increases with age being $17 \%$ in children $<10$ years, $34 \%$ in those aged between $10-15$ years of age and $59 \%$ in patients $>15$ years.

\section{CONCLUSIONS}

- Cardiac manifestations are often found in children with Duchenne muscular dystrophy, representing, after respiratory failure, the most common cause of death.

- In children with Duchenne muscular dystrophy laboratory investigations should include ECG, Holter monitoring, cardiac ultrasound and cardiovascular magnetic resonance.

- Increased sensitivity of cardiovascular magnetic resonance in the early detection of abnormalities or minor changes in myocardial allows to better establish the natural progression of heart damage in Duchenne muscular dystrophy and allows the development of new therapeutic approaches for these patients.

11. Gulati S., Saxena A., Kumaer V. et al. Duchenne muscular dystrophy prevalence and patterns of cardiac involvement. Indian J Pediatr. 2005; 72(5):389-93.

12. Silva M.C., Meira Z.M., Gurgel G.J. et al. Myocardial delayed enhancement by magnetic resonance imaging in patients with muscular dystrophy. J Am Coll Cardiol 2007; 49:1874-9.

13. Puchalski M.D., Williams R.V., Askovich B. et al. Late gadolinium enhancement: precursor to cardiomyopathy in Duchenne muscular dystrophy. Int J Cardiovasc Imaging. 2009; 25:57-63.

14. James J., Kinnett K., Wang Y. et al. Electrocardiographic abnormalities in very young Duchenne muscular dystrophy patients precede the onset of cardiac dysfunction. Neuromuscul Disord. 2011; 21(7):462-7.

15. Takami Y., Takeshima Y., Awano H. et al. High incidence of electrocardiogram abnormalities in young patients with Duchenne muscular dystrophy. Pediatr Neurol. 2008; 39(6):399-403.

16. Mertens L., Ganame J., Claus P. et al. Early regional myocardial dysfunction in young patients with Duchenne muscular dystrophy. J Am Soc Echocardiogr. 2008; 21(9):1049-54.

17. Shabanian R., Aboozari M., Kiani A. Myocardial performance index and atrial ejection force in patients with Duchenne's muscular dystrophy. Echocardiography. 2011; 28(10):1088-94.

18. Lee T.H., Eun Y.E., Choi J.Y. et al. Myocardial atrophy in children with mitochondrial disease and Duchenne muscular dystrophy. Korean J Pediatr. 2014; 57(5):232-239.

19. Florian A. Ludwig A., Rösch S. et al. Myocardial fibrosis imaging based on T1-mapping and extracellular volume fraction (ECV) 
measurement in muscular dystrophy patients: diagnostic value compared with conventional late gadolinium enhancement (LGE) imaging. Eur Heart J Cardiovasc Imaging. 2014; 15(9):1004-12.

20. Hor K.N., Taylor M.D., Al-Khalidi H.R. et al. Prevalence and distribution of late gadolinium enhancement in a large population of patients with Duchenne muscular dystrophy: effect of age and left ventricular systolic function. Journal of Cardiovascular Magnetic Resonance. 2013; 15:107-18.

21. Verhaert D., Richards K., Rafael-Fortney J.A. et al. Cardiac Involvement in Patients with Muscular Dystropies: Magnetic Resonance Imaging Phenotype and Genotypic Considerations. Circ cardiovasc Imaging. 2011; 4:67-76.
22. Wansapura J.P. et al. Left ventricular T2 distribution in Duchenne muscular dystrophy. J Cardiovasc Magn Reson. 2010; 12(1):14.

23. Walcher T. et al. Detection of long-term progression of myocardial fibrosis in Duchenne muscular dystrophy in an affected family: a cardiovascular magnetic resonance study. Eur J Radiol. 2011; 80(1):115-9.

24. Bilchick K.C. et al. Prevalence and distribution of regional scar in dysfunctional myocardial segments in Duchenne muscular dystrophy. J Cardiovasc Magn Reson. 2011; 13:20. 